\title{
Controle glicêmico de pessoas com Diabetes mellitus tipo 2 em uma Unidade de Saúde
}

\section{da Família do interior paulista}

\author{
Glycemic control of people with type 2 Diabetes mellitus in a Family Healthcare Unit in the interior \\ of São Paulo
}

Control glucémico de personas con Diabetes mellitus tipo 2 en una Unidad de Salud de la Familia del interior de São Paulo

Julio Maganha Gouvêa ORCID: https://orcid.org/0000-0001-9027-0722 Faculdade São Leopoldo Mandic, Brasil E-mail:julio.mgouvea@gmail.com

Felipe Biazon Rocha

ORCID: https://orcid.org/0000-0002-4939-5344 Prefeitura Municipal de Araras, Brasil E-mail: felipebiazonrocha@gmail.com

Ivana Daniela Cesar ORCID: https://orcid.org/0000-0001-9199-6381 Faculdade São Leopoldo Mandic, Brasil E-mail: ivana.cesar@slmandic.edu.br

Tássia Fraga Bastos

ORCID: https://orcid.org/0000-0003-0043-9649 Faculdade São Leopoldo Mandic, Brasil E-mail: tassia.bastos@slmandic.edu.br

Elizabeth Regina de Melo Cabral ORCID: https://orcid.org/0000-0002-1903-0993 Faculdade São Leopoldo Mandic, Brasil E-mail: elizabeth.cabral@ slmandicararas.edu.br

Márcio Cristiano de Melo

ORCID: https://orcid.org/0000-0001-9840-0309 Faculdade São Leopoldo Mandic, Brasil

E-mail: marcio.melo@ slmandicararas.edu.br

Bruno Ferrari Emerich

ORCID: https://orcid.org/0000-0001-7246-5809 Faculdade São Leopoldo Mandic, Brasil E-mail: bruno.emerich@slmandicararas.edu.br

Rinaldo Eduardo Machado de Oliveira ORCID: https://orcid.org/0000-0003-1684-1456 Faculdade São Leopoldo Mandic, Brasil

E-mail: rinaldo.oliveira@slmandicararas.edu.br

\begin{abstract}
Resumo
Este estudo objetivou analisar o controle glicêmico de pessoas com Diabetes mellitus tipo 2 em uma Unidade de Saúde da Família do município de Araras, São Paulo. Trata-se de um estudo transversal descritivo baseado na coleta de dados sociodemográficos e clínicos em prontuários físicos. Foram incluídos 102 participantes, sendo que, 66,6\% eram do sexo feminino e média de idade de 61,4 anos. Evidenciou-se que 57,8\% possuíam controle glicêmico inadequado com hemoglobina glicada igual ou superior a 7,0\%. O controle glicêmico inadequado mostrou-se associado a faixa etária, polifarmácia e tipos de medicamentos prescritos para o tratamento do diabetes $(\mathrm{p}<0,05)$. Na farmacoterapia do diabetes, verificou-se a prescrição de um ou dois medicamentos para 72,5\% dos participantes, sendo a metformina prescrita para $80,7 \%$. O estudo mostrou a frequência do controle glicêmico, bem como as variáveis que influenciavam e ressalta a importância da abordagem clínica centrada às pessoas com diabetes na Atenção Primária à Saúde a fỉm de evitar-se complicações decorrentes da doença.
\end{abstract}

Palavras-chave: Diabetes Mellitus; Hiperglicemia; Atenção primária à saúde.

\section{Abstract}

This study aimed to analyze the glycemic control of people with type 2 diabetes mellitus in a Family Healthcare Unit in the city of Araras, São Paulo. This is a descriptive cross-sectional study based on the collection of sociodemographic and clinical data from physical records. 102 participants were included, $66.6 \%$ were female and 
mean age was 61.4 years. It was shown that $57.8 \%$ had inadequate glycemic control with glycated hemoglobin equal to or greater than $7.0 \%$. Inadequate glycemic control was associated with age group, polypharmacy and types of medication prescribed for the treatment of diabetes $(\mathrm{p}<0.05)$. In diabetes pharmacotherapy, one or two medications were prescribed for $72.5 \%$ of the participants, with metformin being prescribed for $80.7 \%$. The study showed the frequency of glycemic control, as well as the variables that influenced it, and highlights the importance of a clinical approach focused on people with diabetes in Primary Health Care in order to avoid complications resulting from the disease.

Keywords: Diabetes Mellitus; Hyperglycemia; Primary health care.

\section{Resumen}

Este estudio tuvo como objetivo analizar el control glucémico de personas con diabetes mellitus tipo 2 en una Unidad de Salud de la Familia de la ciudad de Araras, São Paulo. Se trata de un estudio descriptivo transversal basado en la recogida de datos sociodemográficos y clínicos a partir de registros físicos. Se incluyeron 102 participantes, el 66,6\% eran mujeres y la edad media fue de 61,4 años. Se demostró que el 57,8\% tenía un control glucémico inadecuado con hemoglobina glucosilada igual o superior al 7,0\%. El control glucémico inadecuado se asoció con el grupo de edad, la polifarmacia y el tipo de medicación prescrita para el tratamiento de la diabetes $(\mathrm{p}<0,05)$. En la farmacoterapia de la diabetes, se prescribieron uno o dos medicamentos al 72,5\% de los participantes y metformina al 80,7\%. El estudio mostró la frecuencia del control glucémico, así como las variables que influyeron en él, y destaca la importancia de un abordaje clínico centrado en las personas con diabetes en Atención Primaria de Salud para evitar complicaciones derivadas de la enfermedad.

Palabras clave: Diabetes Mellitus; Hiperglucemia; Atención primaria de salud.

\section{Introdução}

O diabetes mellitus tipo 2 (DM2) é um dos maiores problemas de saúde pública na atualidade. A Organização Mundial da Saúde estima que a glicemia elevada é o terceiro fator, em importância, da causa de mortalidade prematura, superada apenas por hipertensão arterial sistêmica e o uso de tabaco (WHO, 2009).

A fisiopatologia do DM2 é caracterizada pela resistência insulínica que ocorre pela insensibilidade da insulina, depleção da produção deste hormônio e uma possível falha das células beta nas ilhotas pancreáticas (ADA, 2020; SBD, 2019). Essas desordens geram uma diminuição do transporte de glicose para os tecidos, associada à decomposição de gordura com hiperglicemia (Fujioka, 2007).

A Federação Internacional de Diabetes estimou, em 2017, que cerca de 8,8\% da população mundial com idade compreendida entre 20 e 79 anos, vivia com diabetes. Os dados são preocupantes e mostraram que, caso este padrão persista, em 2045, haverá mais de 628,6 milhões de pessoas com a doença (IDF, 2019).

No Brasil, a pesquisa de vigilância de fatores de risco e proteção para doenças crônicas por inquérito telefônico, de 2018, realizada nas capitais brasileiras e no distrito federal estimou a prevalência de diabetes em 7,7\%. Em 2006, a prevalência estimada foi de 5,5\%. Logo, percebeu-se um aumento de 60\% no período considerado (Ministério da Saúde, 2019).

O diabetes é uma condição sensível à Atenção Primária à Saúde (APS). Logo, destaca-se que o bom manejo desse agravo no nível primário de atenção evita hospitalizações, bem como, mortes por complicações cardiovasculares e cerebrovasculares. Logo, a APS deve atuar como porta de entrada no sistema de saúde e coordenadora do cuidado para que as ações voltadas ao controle do diabetes desenvolvam-se de forma efetiva e eficiente (Borges \& Lacerda, 2018).

Os estudos brasileiros explicitaram que, na APS ainda há predomínio de uma assistência voltada para a demanda de consultas médicas, sem que tal ação fosse embasada pela política de saúde vigente às pessoas com doenças crônicas não transmissíveis (Borges \& Lacerda, 2018; Salci, et al., 2017). Contudo, experiências exitosas mostraram que o controle glicêmico é melhor obtido empregando-se estratégias baseadas no método clínico centrado na pessoa, considerando as subjetividades dos indivíduos, bem como, o desenvolvimento de ações educativas (Edelman \& Polonsky, 2017; Ministério da Saúde, 2013).

Na APS, recomenda-se um esforço contínuo para o acompanhamento e tratamento das pessoas com DM2 em consonância com as definições de cuidado integral e longitudinal do diabetes, incluindo o estímulo às mudanças de estilo de 
vida (MEV), o controle glicêmico e metabólico, bem como, a prevenção das complicações crônicas. As MEV para hábitos mais saudáveis é a base do tratamento do diabetes e pode contribuir para o controle de outros fatores de risco para doenças cardiovasculares (Goes, et al., 2020).

$\mathrm{O}$ atual tratamento preconizado do DM2 inclui as MEV, uso de medicamentos e educação em diabetes. A prevenção primária se estabelece a partir de uma proposta alimentar saudável e exercício físico regular que postergam as necessidades de atenção à doença. A prevenção secundária inclui diagnóstico precoce e tratamento adequado, abrangendo o controle dos níveis glicêmicos e de colesterol, podendo assim reduzir substancialmente o risco das complicações da doença e sua progressão (Malta, et al., 2019).

Em Unidades de Saúde da Família (USF) do interior paulista há escassez de dados relacionados à gestão do cuidado e assistência do DM2. Neste contexto, o conhecimento científico acerca do tema ganha relevância, uma vez que, a ausência do manejo clínico adequado representa um forte preditor de desfechos desfavoráveis. A realização de estudos capazes de retratar um panorama clínico e epidemiológico em serviços específicos da APS é uma estratégia importante para a compreensão, reflexão e produção de evidências para a comunidade acadêmica, gestores e profissionais de saúde. Assim, o objetivo deste estudo foi analisar o controle glicêmico de pessoas com DM2 em uma USF do município de Araras, São Paulo (SP).

\section{Metodologia}

Trata-se de um estudo transversal descritivo baseado em informações coletadas na revisão de prontuários físicos de uma USF do município de Araras, SP. Incluíram-se na pesquisa o prontuário das pessoas com diagnóstico médico de DM2 cadastradas na USF, idade igual ou superior a 25 anos e ambos os sexos. Foram excluídos os prontuários das pessoas que eram cadastradas na USF, possuíam diagnóstico médico de DM2, mas não realizaram consulta médica na USF para acompanhamento do DM2 no ano de 2019.

$\mathrm{Na}$ USF havia 2.896 pessoas cadastradas no período de planejamento do estudo (maio de 2020), sendo que 338 possuíam o diagnóstico médico de DM2. Assim, no cálculo da amostra considerou-se a prevalência estimada de DM2 de $11,6 \%$. Optou-se por um erro absoluto tolerável de $5 \%$ e coeficiente de confiança de $95 \%$. A partir destes dados, o tamanho da amostra foi estimado em 102 prontuários. No início do estudo, foi obtida a relação completa das pessoas com DM2 e os prontuários foram alcançados por meio de amostragem aleatória simples. Caso os critérios de inclusão não fossem preenchidos, realizava-se um novo sorteio.

A coleta de dados ocorreu no período de novembro de 2020 a abril de 2021, tendo como base o registro da última consulta médica realizada no ano de 2019. O formulário elaborado pelos pesquisadores incluiu as seguintes variáveis: sexo, idade, cor/raça, escolaridade, peso, altura, uso de bebida alcóolica, tabaco, tempo de diagnóstico do DM2, realização do exame do pé diabético no ano de 2019, encaminhamento ao oftalmologista no ano de 2019, doenças associadas ao diabetes, medicamentos prescritos, exames laboratoriais e participação em ações coletivas de educação em diabetes.

A variável dependente foi o controle glicêmico categorizada conforme a Sociedade Brasileira de Diabetes (SBD). Os prontuários dos participantes com hemoglobina glicada (HbA1c) menor que 7,0\% foram consideradas pessoas com DM2 com controle glicêmico adequado e aqueles com HbA1c maior ou igual a 7,0\% com controle glicêmico inadequado.

No índice de massa corporal considerou-se eutrofia e baixo peso $\left(<25 \mathrm{~kg} / \mathrm{m}^{2}\right)$, sobrepeso (entre 25 e $\left.29,9 \mathrm{~kg} / \mathrm{m}^{2}\right)$ e obesidade $\left(\geq 30 \mathrm{~kg} / \mathrm{m}^{2}\right)$ (OMS, 2000). Já a polifarmácia foi definida como cinco ou mais medicamentos prescritos (Secoli, 2010).

Realizou-se um estudo piloto com dez prontuários, para verificar a aplicabilidade do formulário e a logística do trabalho de campo. Os prontuários analisados nesta etapa não foram incluídos na amostra final do estudo, pois, verificou-se carência de dados acerca da cor/raça, escolaridade, uso de bebida alcoólica, tabaco, tempo de diagnóstico do DM2, realização 
do exame do pé diabético no ano de 2019, encaminhamento ao oftalmologista no ano de 2019 e participação em ações coletivas de educação em diabetes. Assim, tais variáveis foram excluídas da versão final do formulário.

Os dados foram armazenados em um banco de dados específico do Excel e analisados por meio do software R. Na descrição dos dados foram utilizadas medidas de tendência central e de dispersão para variáveis quantitativas e porcentagens para variáveis qualitativas. A comparação entre variáveis categóricas foi feita pelo teste do qui-quadrado de Pearson e o nível de significância adotado foi de $5 \%$.

O estudo obteve aprovação do Comitê de Ética em Pesquisa da Faculdade São Leopoldo Mandic com CAAE n ${ }^{\circ}$ 37201520.6.0000.5374 e parecer $\mathrm{n}^{\mathrm{o}}$ 4.288.196.

\section{Resultados}

Dos 102 prontuários analisados, 66,6\% eram do sexo feminino. A idade variou de 34 a 80 anos com média de 61,4 anos ( $\mathrm{DP}=9,4)$, sendo 60,8\% com idade igual ou superior a 60 anos. Quanto ao número de doenças, 80,4\% possuíam mais de três doenças sendo que o sobrepeso/obesidade esteve presente em 84,3\%, seguido da hipertensão arterial sistêmica $(68,6 \%)$ e dislipidemia (20,6\%). A polifarmácia mostrou-se frequente em 73,5\% dos participantes. No tratamento do DM2, observou-se a prescrição de um ou dois medicamentos para a maioria dos participantes (Tabela 1).

Tabela 1. Características sociodemográficas, clínicas e farmacoterapêuticas das pessoas com diabetes mellitus tipo 2 de uma Unidade de Saúde da Família. Araras, São Paulo, 2021. (n=102).

\begin{tabular}{lcc}
\hline Variáveis & $\mathbf{n}$ & $\mathbf{\%}$ \\
\hline Sexo & 68 & 66,6 \\
Feminino & 34 & 33,4 \\
Masculino & $61,4(9,4)$ & - \\
Média de idade (DP) (anos) & 20 & 19,6 \\
Número de doenças & 43 & 42,1 \\
$1-2$ & 39 & 38,3 \\
$3-4$ & & \\
$\geq 5$ & 86 & 84,3 \\
Principais doenças associadas ao diabetes & 70 & 68,6 \\
Sobrepeso/obesidade & 21 & 20,6 \\
Hipertensão Arterial Sistêmica & 20 & 19,6 \\
Dislipidemia & 19 & 18,6 \\
Depressão & 14 & 13,7 \\
Doenças oftalmológicas & 11 & 10,8 \\
Hiperuricemia & & \\
Doenças da tireoide & 27 & 26,5 \\
Número de medicamentos utilizados & 42 & 41,2 \\
$1-4$ & 33 & 32,3 \\
$5-8$ & & \\
$\geq 9$ & & 72,5 \\
Número de medicamentos utilizados para o tratamento do & 74 & 27,5 \\
diabetes & 28 & \\
$1-2$ & & \\
$3-4$ &
\end{tabular}

Fonte: Autores.

A metformina esteve prescrita em monoterapia para 36,3\% dos participantes e 5,9\% não havia medicamentos prescritos para o tratamento do DM2 (Tabela 2). As sulfonilureias prescritas nos prontuários analisados foram: gliclazida (47\%), glibenclamida $(5,8 \%)$ e glimepirida $(0,9 \%)$. Dentre os medicamentos prescritos para as outras doenças destacaram-se: losartana (48\%), hidroclorotiazida $(28,4 \%)$ e omeprazol $(28,4)$. 
Tabela 2. Medicamentos prescritos para as pessoas com diabetes mellitus tipo 2 de uma Unidade de Saúde da Família. Araras, São Paulo, 2021. $(\mathrm{n}=102)$.

\begin{tabular}{lcc}
\hline Medicamentos & $\mathbf{n}$ & \% \\
\hline Sem medicamentos prescritos & 6 & 5,9 \\
Apenas metformina & 37 & 36,3 \\
Apenas sulfonilureia & 2 & 1,9 \\
Metformina e sulfonilureia & 30 & 29,4 \\
Apenas insulina(s) & 2 & 1,9 \\
Insulina(s) e antidiabético oral & 25 & 24,6 \\
\hline Total & $\mathbf{1 0 2}$ & $\mathbf{1 0 0}$ \\
\hline
\end{tabular}

Fonte: Autores.

A média dos resultados do exame laboratorial de glicemia de jejum foi de 147,4 mg/dL (DP=30,3). Os resultados laboratoriais de $\mathrm{HbA1c}$ variam de 5,1\% a 15,3\% com média de 7,9\% (DP=2,9). Do total de participantes, 59 $(57,8 \%)$ apresentaram HbA1c igual ou superior a 7,0\%, evidenciado controle glicêmico inadequado. Mostraram-se associados ao controle glicêmico inadequado a faixa etária, polifarmácia e tipos de medicamentos prescritos para o tratamento do diabetes (Tabela 3).

Tabela 3. Variáveis sociodemográficas, clínicas e farmacoterapêuticas das pessoas com diabetes mellitus tipo 2 segundo o controle glicêmico inadequado de uma Unidade de Saúde da Família. Araras, São Paulo, 2021. (n=59)

\begin{tabular}{|c|c|c|c|}
\hline \multirow[t]{2}{*}{ Variáveis } & \multicolumn{2}{|c|}{ Controle glicêmico inadequado $(\mathrm{HbA1c} \geq 7,0 \%)$} & \multirow[t]{2}{*}{ p-valor* } \\
\hline & $\mathbf{n}$ & $\%$ & \\
\hline \multicolumn{4}{|l|}{ Sexo } \\
\hline Feminino & 41 & 69,5 & 0,47 \\
\hline Masculino & 18 & 30,5 & \\
\hline \multicolumn{4}{|l|}{ Faixa etária (anos) } \\
\hline$<60$ & 26 & 44,1 & $\mathbf{0 , 0 2}$ \\
\hline$\geq 60$ & 33 & 55,9 & \\
\hline \multicolumn{4}{|l|}{ Número de doenças } \\
\hline $1-2$ & 14 & 23,7 & 0,08 \\
\hline$\geq 3$ & 45 & 76,3 & \\
\hline \multicolumn{4}{|l|}{ Polifarmácia* } \\
\hline Sim & 13 & 22,0 & $<0,01$ \\
\hline Não & 46 & 78,0 & \\
\hline \multicolumn{4}{|c|}{ Medicamentos prescritos para o diabetes } \\
\hline Sim & 56 & 94,9 & 0,68 \\
\hline Não & 3 & 5,1 & \\
\hline \multicolumn{4}{|l|}{ Tipos de medicamentos } \\
\hline Antidiabético oral & 31 & 52,6 & $<0,01$ \\
\hline Insulina(s) & 1 & 1,7 & \\
\hline Antidiabético oral e insulina & 24 & 40,7 & \\
\hline
\end{tabular}

*Teste do qui-quadrado de Pearson. **Cinco ou mais medicamentos prescritos (Secoli, 2010). Fonte: Autores.

\section{Discussão}

No presente estudo, verificou-se que mais de $50 \%$ dos participantes mostraram controle glicêmico inadequado, sendo influenciado pela faixa etária, polifarmácia e tipo de medicamento utilizado no tratamento do DM2. A elevada frequência apontou-se semelhante a outros estudos brasileiros realizados na APS. Em uma pesquisa conduzida na Estratégia Saúde da Família (ESF) de Pernambuco com 787 pessoas com DM2 evidenciou que mais de 65\% apresentaram HbA1c igual ou maior a 7,0\%. O controle glicêmico inadequado esteve associado aqueles com idade menor, duração da doença mais longa, mais contatos anuais com a ESF e regime terapêutico complexo (Lima, et al., 2016).

No estudo longitudinal de saúde do adulto, observou-se que em 54,2\% dos participantes com DM autorreferido a 
$\mathrm{HbA1c}$ era igual ou maior a 6,5\%. Além disso, o controle glicêmico inadequado esteve associado ao sexo masculino, cor/raça da pele preta ou parda, nível médio de ocupação, não possuir plano privado de saúde, usar insulina, relação cintura-quadril alterada, tabagismo e autoavaliação da saúde sendo ruim ou muito ruim (Moraes, et al., 2020).

A maioria dos participantes desta pesquisa era do sexo feminino, possuía idade igual ou superior a sessenta anos, diagnóstico de duas ou mais doença e havia prescrição de cinco ou mais medicamentos. Este perfil de participantes pode ser justificado pela maior procura das mulheres aos serviços da APS, criação de vínculos com a equipe e maior autocuidado em diabetes (Oliveira, et al., 2017). Ademais, ressalta-se que o DM2 acomete frequentemente as pessoas acima de trinta anos e aumenta com a idade (Malta, et al., 2019). O elevado número de doenças associadas ao diabetes e a polifarmácia traz uma demanda à USF relacionada a abordagem multiprofissional à prevenção de agudizações, efeitos vasculares e sistêmicos (Prado, et al., 2016).

$\mathrm{O}$ número de participantes com sobrepeso ou obesidade mostrou-se preocupante. $\mathrm{O}$ consumo de carboidratos em excesso e a inatividade física podem contribuir para o acúmulo de gordura corporal, além de ocasionar resistência à insulina. Na APS a integralidade do cuidado às pessoas com diabetes deve privilegiar uma abordagem que não seja exclusivamente focada na terapia medicamentosa, mas que valorize as MEV (Baldoni \& dal Fabbro, 2018). As equipes da ESF em conjunto com o apoio matricial do Núcleo Ampliado de Saúde da Família devem organizar os processos de trabalho atendo-se às necessidades das pessoas com diabetes (Bezerra, et al., 2020).

Quanto aos medicamentos para o diabetes, a American Diabetes Association (ADA) e a SBD recomendam a prescrição no diagnóstico da doença, uma vez que é essencial para o tratamento (ADA, 2020; SBD, 2019). Contudo, no presente estudo verificou-se que 5,9\% dos prontuários analisados não havia medicamentos prescritos. A metformina foi o principal antidiabético oral (ADO) prescrito. A indicação em monoterapia corresponde a primeira linha do tratamento (ADA, 2020; SBD, 2019). Entretanto, percebeu-se que as pessoas com prescrição de ADO e insulina(s) apresentaram controle glicêmico adequado. Assim, ressalta-se que a educação continuada aos médicos da APS é fundamental para a atualização das condutas clínicas, bem como, para execução dos projetos terapêuticos singulares.

Um estudo realizado com pessoas com DM2 na ESF de Itajaí, Santa Catarina apontou que organização da atenção às pessoas seguindo os atributos da integralidade, longitudinalidade e coordenação do cuidado, poderia interferir de forma positiva no autocuidado nos aspectos que dificultavam o controle glicêmico. No presente estudo, não foi possível analisar o controle glicêmico e a relação com os atributos da APS. Contudo, enfatiza-se que a multiplicidade da atenção ao diabetes deve permear os programas assistenciais de cuidado (Maeyama, 2020).

Por fim, cita-se uma revisão sistemática com meta-análise que mostrou que o empoderamento coletivo das pessoas com DM apresentou melhoria dos parâmetros clínicos, comportamentais e aumentou o conhecimento sobre o DM e autocuidado. Assim, enfatiza-se que a APS sendo a porta de entrada aos serviços de saúde deve analisar as multidimensões para o cuidado integral (Baldoni, et al., 2017). Em períodos que se permite a reunião de pessoas, as intervenções coletivas em diabetes mostram-se promissoras e devem integrar as agendas das USF. Ademais, o emprego de tecnologias da informação e comunicação, dentre elas a telemedicina, são instrumentos que podem favorecer o controle glicêmico às pessoas com DM (Timpel, et al., 2020).

\section{Considerações Finais}

Este estudo mostrou a frequência do controle glicêmico, bem como as variáveis que influenciavam por meio da análise de prontuários físicos em uma USF do interior paulista. Dentre as limitações destaca-se a ausência do registro de dados sociodemográficos, clínicos e terapêuticos, que inclui as orientações acerca da alimentação, prática de exercícios físicos, bem como, o seguimento em outros serviços da Rede de Atenção à Saúde. 
Ressalta-se que a assistência ao DM2 na APS é complexa, envolve o controle glicêmico e os aspectos biopsicossociais relacionados à doença. Diante do exposto, as evidências aqui elencadas trazem subsídios para a qualificação do cuidado às pessoas com diabetes e longitudinalidade no contexto do estudo.

\section{Referências}

American Diabetes Association. (2020). Standards of Medical Care in Diabetes-2020 (43A ed.). Clinical Diabetes. https://www.ncbi.nlm.nih.gov/pmc/articles/PMC6969656/

Baldoni, N. R., \& dal Fabbro, A. L. (2018). Nutritional profile of patients with type 2 Diabetes Mellitus in Ribeirão Preto family health units. Ciência \& Saúde, 11(2), 121. https://doi.org/10.15448/1983-652x.2018.2.26030

Baldoni, N. R., Aquino, J. A., Sanches-Giraud, C., di Lorenzo Oliveira, C., de Figueiredo, R. C., Cardoso, C. S., Santos, T. R., Alves, G. C. S., dal Fabbro, A. L., \& Baldoni, A. O. (2017). Collective empowerment strategies for patients with Diabetes Mellitus: A systematic review and meta-analysis. Primary Care Diabetes, 11(2), 201-211. https://doi.org/10.1016/j.pcd.2016.09.006

Bezerra, H. M. D. C., Gomes, M. F., Oliveira, S. R. D. A., \& Cesse, E. N. P. (2020). Processo educativo do núcleo ampliado de saúde da família na atenção à hipertensão e diabetes. Trabalho, Educação e Saúde, 18(3). https://doi.org/10.1590/1981-7746-sol00277

Borges, D. B., \& Lacerda, J. T. (2018). Ações voltadas ao controle do Diabetes Mellitus na Atenção Básica: proposta de modelo avaliativo. Saúde Debate, 42(116), 162-178. https://www.scielo.br/j/sdeb/a/Rw6pYJ7C9PVwdCpYBYfp5yh/?lang=pt

Edelman, S. V., \& Polonsky, W. H. (2017). Type 2 Diabetes in the Real World: The Elusive Nature of Glycemic Control. Diabetes Care, 40(11), 1425-1432. https://doi.org/10.2337/dc16-1974

Fujioka K. (2007). Pathophysiology of type 2 diabetes and the role of incretin hormones and beta-cell dysfunction. JAAPA : official journal of the American Academy of Physician Assistants, Suppl, 3-8. https://doi.org/10.1097/01720610-200712000-00001

International Diabetes Federation. (2019). IDF Atlas (9th ed.). https://diabetesatlas.org/en/

Lima, R. F., Fontbonne, A., Carvalho, E. M. F., Montarroyos, U. R., Barreto, M. N. S. C., \& Cesse, E. A. P. (2016). Fatores associados ao controle glicêmico em pessoas com diabetes na Estratégia Saúde da Família em Pernambuco. Revista Da Escola de Enfermagem Da USP, 50(06), 937-945. https://www.scielo.br/j/reeusp/a/CXkCkjzRB44CRZQK5MxbQ7c/?lang=pt\#

Maeyama, M. A., Pollheim, L. C. F., Wippel, M., Machado, C., \& Veiga, M. V. (2020). Aspectos relacionados à dificuldade do controle glicêmico em pacientes com diabetes mellitus tipo 2 na Atenção Básica. Brazilian Journal of Development, 6(7), 47352-47369. https://doi.org/10.34117/bjdv6n7-391

Malta, D. C., Andrade, S. S. C. D. A., Oliveira, T. P., Moura, L. D., Prado, R. R. D., \& Souza, M. D. F. M. D. (2019). Probabilidade de morte prematura por doenças crônicas não transmissíveis, Brasil e regiões, projeções para 2025. Revista Brasileira de Epidemiologia, 22. https://doi.org/10.1590/1980549720190030

Malta, D. C., Duncan, B. B., Schmidt, M. I., Machado, S. E., Silva, A. G. D., Bernal, R. T. I., Pereira, C. A., Damacena, G. N., Stopa, S. R., Rosenfeld, L. G., \& Szwarcwald, C. L. (2019). Prevalência de diabetes mellitus determinada pela hemoglobina glicada na população adulta brasileira, Pesquisa Nacional de Saúde. Revista Brasileira de Epidemiologia, 22(suppl 2). https://doi.org/10.1590/1980-549720190006.supl.2

Ministério da Saúde. (2013). Estratégias para o cuidado da pessoa com doenças crônicas: diabetes mellitus. https://bvsms.saude.gov.br/bvs/publicacoes/estrategias_cuidado_pessoa_diabetes_mellitus_cab36.pdf

Ministério da Saúde. (2019). Vigitel Brasil 2018. https://portalarquivos2.saude.gov.br/images/pdf/2019/julho/25/vigitel-brasil-2018.pdf

Moraes, H. A. B. D., Mengue, S. S., Molina, M. D. C. B., \& Cade, N. V. (2020). Fatores associados ao controle glicêmico em amostra de indivíduos com diabetes mellitus do Estudo Longitudinal de Saúde do Adulto, Brasil, 2008 a 2010. Epidemiologia e Serviços de Saúde, 29(3). https://doi.org/10.5123/s167949742020000300017

Oliveira, R. E. M., Baldoni, N. R., Ueta, J., \& Franco, L. J. (2017). Diferenças de gênero na utilização dos serviços de saúde por indivíduos com diabetes mellitus tipo 2. Espaço Para a Saúde - Revista de Saúde Pública do Paraná, 18(1), 100. https://doi.org/10.22421/1517-7130.2017v18n1p100

Prado, M. A. M. B. D., Francisco, P. M. S. B., \& Barros, M. B. D. A. (2016). Diabetes em idosos: uso de medicamentos e risco de interação medicamentosa. Ciência \& Saúde Coletiva, 21(11), 3447-3458. https://doi.org/10.1590/1413-812320152111.24462015

Salci, M. A., Meirelles, B. H. S., \& Silva, D. M. G. V. D. (2017). Primary care for diabetes mellitus patients from the perspective of the care model for chronic conditions. Revista Latino-Americana de Enfermagem, 25(0). https://doi.org/10.1590/1518-8345.1474.2882

Secoli, S. R. (2010). Polifarmácia: interações e reações adversas no uso de medicamentos por idosos. Revista Brasileira de Enfermagem, 63(1), 136-140. https://doi.org/10.1590/s0034-71672010000100023

Sociedade Brasileira de Diabetes (2019). Diretrizes da Sociedade Brasileira de Diabetes 2019/2020. https://diabetes.org.br/profissionais/images/DIRETRIZESCOMPLETA-2019-2020.pdf

Timpel, P., Oswald, S., Schwarz, P. E. H., \& Harst, L. (2020). Mapping the Evidence on the Effectiveness of Telemedicine Interventions in Diabetes, Dyslipidemia, and Hypertension: An Umbrella Review of Systematic Reviews and Meta-Analyses. Journal of Medical Internet Research, 22(3), e16791. https://doi.org/10.2196/16791 
Research, Society and Development, v. 10, n. 11, e588101119982, 2021

(CC BY 4.0) | ISSN 2525-3409 | DOI: http://dx.doi.org/10.33448/rsd-v10i11.19982

World Health Organization. (2000). Obesity: preventing and managing the global epidemic: report of a WHO consultation. https://apps.who.int/iris/handle/10665/42330

World Health Organization. (2009). Global health risks: mortality and burden of disease attributable to selected major risks. Department of Health Statistics and Informatics in the Information, Evidence and Research Cluster of the World Health Organization. https://apps.who.int/iris/bitstream/handle/10665/44203/9789241563871_eng.pdf?sequence=1\&isAllowed=y 